\title{
MARCH8 Restricts Ebola Virus Replication by Blocking the Viral Glycoprotein Processing and Glycosylation +
}

\author{
Changqing Yu 1, Sunan Li ${ }^{1}$, Omid Madadgar ${ }^{2}$, Iqbal Ahmad 1, Xianfeng Zhang ${ }^{3}$, Jing Shi 1, \\ Yu Wang ${ }^{4}$, Yulong Zhou ${ }^{5}$ and Yong-Hui Zheng 1,2,* \\ 1 Harbin Veterinary Research Institute, CAAS-Michigan State University Joint Laboratory of Innate \\ Immunity, State Key Laboratory of Veterinary Biotechnology, Chinese Academy of Agricultural Sciences, \\ Harbin 150069, China; ycq_1926@126.com (C.Y.); lsnlhh2006@163.com (S.L.); \\ iqbal_microbiologist@yahoo.com (I.A.); shijing@westlake.edu.cn (J.S) \\ 2 Department of Microbiology and Molecular Genetics, Michigan State University, East Lansing, MI 48824, \\ USA; madadgar@msu.edu \\ 3 National High Containment Facilities for Animal Diseases Control and Prevention, State Key Laboratory of \\ Veterinary Biotechnology, Chinese Academy of Agricultural Sciences, Harbin 150069, China; \\ zhangxianfeng@CAAS.cn \\ 4 Laboratory of Core facility, State Key Laboratory of Veterinary Biotechnology, Chinese Academy of \\ Agricultural Sciences, Harbin 150069, China; yuiop6183@163.com \\ 5 College of Animal Science and Technology, Heilongjiang Bayi Agricultural University, Daqing 10223, \\ China; zhouyulong1980@163.com \\ * Correspondence: zhengyo@msu.edu \\ † Presented at Viruses 2020-Novel Concepts in Virology, 5-7 February 2020. \\ Published: 7 July 2020
}

\begin{abstract}
Ebola virus (EBOV) glycoprotein (GP) is a class I fusion protein whose maturation is dependent on furin-mediated processing. EBOV-GP is heavily glycosylated, with glycans constituting $\sim 50 \%$ of its molecular mass. Compared with $15 \mathrm{~N}$-linked glycosylation sites, EBOV-GP1 has $\sim 80$ potential $O$-linked glycosylation sites in the mucin-like domain (MLD), suggesting that $O$ linked glycans are dominated. The membrane-associated RING-CH (MARCH) family consists of 11 members that are RING-finger ubiquitin E3 ligases. Recently, human MARCH1, MARCH2, and MARCH8 were reported to inhibit HIV-1 replication by targeting its Env. Here, we show that human MARCH8 also inhibits EBOV replication by blocking GP incorporation into virions via downregulating its cell surface expression. To understand how the downregulation occurs, we investigated EBOV-GP subcellular localization, processing, glycosylation, and intracellular trafficking in the presence of human MARCH8. We find that MARCH8 interacts with GP and retains GP in the Golgi. MARCH8 also interacts with the homoB domain of furin that blocks its convertase activity. In consequence, MARCH8 blocks GP processing in an MLD-independent manner. Consistently, MARCH8 also blocks the O-linked, but not the $N$-linked glycosylation of GP. Importantly, in the presence of MARCH8, the shedding of GP1 but not the secreted GP (sGP) is blocked, suggesting that MARCH8 targets the GP1 C-terminal region. The MARCH8 activity is extended to its orthologs from Bos taurus and mice, and its paralogs MARCH1 and MARCH2. In addition, MARCH8 inhibits the processing of two other class I fusion proteins, including HIV-1 Env and IAV HA, and it triggers the degradation of the class III fusion protein VSV-G. We conclude that MARCH8 exerts a very broad and conserved antiviral activity by inhibiting the maturation of class I fusion proteins, which blocks their secretion to the cell surface and incorporation into virions. It should also target class III fusion proteins by triggering their degradation.
\end{abstract}


Keywords: MARCH; class I fusion; maturation; ebola; HIV; influenza

(c) (P)

(C) 2020 by the authors. Licensee MDPI, Basel, Switzerland. This article is an open access article distributed under the terms and conditions of the Creative Commons Attribution (CC BY) license (http://creativecommons.org/licenses/by/4.0/). 